\title{
Tilly-la-Campagne (Calvados) « Chemin RN 158 »
}

Nouveaux témoins d'occupation du Néolithique ancien

Tilly-la-Campagne (Calvados) "Chemin RN 158". New evidence of Early Neolithic occupation

Tilly-la-Campagne (Calvados, Normandie, France) „Chemin RN 158“. Neue

Hinweise auf eine Besiedlung im Frühneolithikum

Tilly-la-Campagne (Calvados) "Camino RN 158". Nuevos testigos de ocupación temprana Neolítico

François Charraud, Pierre Giraud, Denis Jan, Anna Baudry et Cécile Germain-Vallée

\section{CpenEdition}

\section{Journals}

Édition électronique

URL : https://journals.openedition.org/rao/3258

DOI : 10.4000/rao.3258

ISSN : 1775-3732

Éditeur

Presses universitaires de Rennes

\section{Édition imprimée}

Date de publication : 31 décembre 2016

Pagination : $39-53$

ISBN : 978-2-7535-5683-6

ISSN : 0767-709X

\section{Référence électronique}

François Charraud, Pierre Giraud, Denis Jan, Anna Baudry et Cécile Germain-Vallée, «Tilly-la-

Campagne (Calvados) « Chemin RN 158 » , Revue archéologique de l'Ouest [En ligne], 33 | 2016, mis en ligne le 31 juillet 2019, consulté le 22 août 2022. URL : http://journals.openedition.org/rao/3258 ; DOI : https://doi.org/10.4000/rao.3258 


\title{
Tilly-la-Campagne (Calvados) "Chemin RN 158 ", nouveaux témoins d'occupation du Néolithique ancien
}

\author{
Tilly-la-Campagne (Calvados) "Chemin RN 158", \\ New Evidence of Early Neolithic Occupation
}

\author{
François Charraud ${ }^{\mathrm{a}}$, Pierre Giraud ${ }^{\mathrm{b}}$, Denis Jan ${ }^{\mathrm{c}}$ et Anna Baudry ${ }^{\mathrm{d}}$, \\ avec la collaboration de Cécile Germain-VALLÉE ${ }^{b}$
}

\begin{abstract}
Résumé : Un diagnostic préventif réalisé par le service archéologique du conseil général du Calvados, en 2007 à Tilly-la-Campagne (Calvados) a mis en évidence une structure isolée du Néolithique ancien (St. 72). Il s'agit d'une fosse à deux lobes peu profonds creusés dans le lœss, au contour irrégulier, orientée est-ouest, oblongue et mesurant 4,40 mètres par 2,15 mètres. Le mobilier mis au jour, principalement dans la partie est de la fosse, est peu abondant mais varié. Il comprend du mobilier en silex (587 pièces), des fragments de poteries (199 tessons), quelques fragments d'outils de mouture lithiques (14 éléments), des fragments d'anneaux en schiste (4 parures), des nodules d'hématite oolithique (5 éléments), des restes fauniques (229 restes) et des coquilles de noisettes carbonisées. Une datation radio-isotopique a été tentée sur ces dernières, sans livrer de résultat exploitable. Cette structure est vraisemblablement tout ce qui subsiste d'un site probablement plus étendu, arasé par les importantes activités agricoles de la Plaine de Caen. Les quelques éléments recueillis permettent de supposer que l'on a affaire à un site à vocation domestique. Par sa nature et la diversité du mobilier qu'elle contenait, cette fosse évoque celles qui bordent de nombreuses architectures domestiques documentées pour le Néolithique ancien. La céramique comporte d'ailleurs comme principaux marqueurs culturels deux tessons décorés d’impressions au peigne, technique largement employée par les groupes culturels de tradition danubienne. La présence d'un fragment d'anneau à couronne large et d'un tranchet pourrait renvoyer au Blicquy/Villeneuve-Saint-Germain récent.
\end{abstract}

Abstract: A preventive operation conducted by the Archaeological Service of the Calvados Council in 2007 in Tilly-la-Campagne (Calvados, revealed an isolated feature dating to the Early Neolithic (St. 72). This two lobed pit dug into the loess is an irregular elongated shape, oriented east-west and measuring $4.40 \mathrm{~m}$ by $2.15 \mathrm{~m}$. The sparse but diverse finds were mainly discovered in the eastern part of the pit. They include worked fint (587 pieces), pottery (199 sherds), fragments of milling tools (14 items), fragments of schist rings (4 sets), hematite nodules (5 items), animal bone (229 residues) and charred hazelnut shells. The attempted radiocarbon analysis did not give a usable result. This structure is probably all that remains of a possibly larger site, destroyed by the agricultural activities of the Caen plain. The nature and diversity of the finds indicate an Early Neolithic domestic site. The pottery is also a prominent cultural marker with two sherds decorated with impressions, technique widely used by Danubian cultural groups. The presence of a large ring fragment and a tranchet could also refer to the end of Blicquy/Villeneuve-Saint-Germain.

Mots clés : Néolithique ancien, Normandie, Calvados, Plaine de Caen, Villeneuve-Saint-Germain, habitat, fosse, silex, parure en schiste, céramique, faune, hématite, macro outillage.

Keywords: Early Neolithic, Normandy, Calvados, Caen Plain, Villeneuve-Saint-Germain, settlement, ditches, schist bangle, pottery, flint, macro tools.

\footnotetext{
${ }^{a}$ Contractuel au conseil général du Calvados et à l'Inrap - Service Archéologie - 36 rue Fred-Scamaroni 14000 CAEN. (fcharraud@gmail.com)

${ }^{b}$ Conseil général du Calvados - Service Archéologie - DGA Jeunesse, Culture et territoires, 36 rue Fred-Scamaroni 14000 CAEN. (pierre.giraud@ calvados.fr)

c Contractuel au conseil général du Calvados et à l'Inrap, 39 boulevard Winston-Churchill, 14100 LISIEUX. (jan.denis@ymail.com)

'Inrap GSO, UMR 6566 CReAAH - Centre archéologique de Poitiers, 122 rue de la Bugellerie, 86000 POITIERS. (anna.baudry-dautry@inrap.fr)
} 


\section{INTRODUCTION}

Le site de Tilly-la-Campagne se trouve à huit kilomètres au sud de l'agglomération caennaise, dans une zone de plaine limoneuse en grande partie mise en culture, à une altitude de 80 m NGF. Il est localisé à moins de cinq kilomètres du cours de l'Orne et à moins de six kilomètres de son affluent la Laize (fig. 1). Il a été découvert en 2007 à l'occasion d'un diagnostic archéologique réalisé par le service archéologie du conseil général du Calvados, sur l'emprise d'un chemin de huit mètres de largeur et d'un peu plus de quatre cents mètres de longueur, situé le long de la route de Falaise (RN 158).

Les vestiges mis au jour couvrent diverses périodes avec des fossés parcellaires et/ou d'enclos, des carrières de calcaire et quelques «trous d'homme » de la seconde guerre mondiale. La structure la plus remarquable est une unique fosse attribuée au Néolithique ancien (St. 72). Cette structure a été entièrement décapée et fouillée dans le cadre du diagnostic archéologique. Une fenêtre d'environ cinquante mètres carrés a également été ouverte autour de la fosse, dans l'emprise du projet routier, sans qu'aucune autre structure néolithique n'y soit observée. La fosse 72 a été fouillée en quatre zones (A, B, C, D) et par passes de $5 \mathrm{~cm}$ de profondeur.

Il s'agit d'une unique fosse fouillée finement avec une stratigraphie lisible et différents types de mobilier. Elle offre un point d'ancrage supplémentaire pour contribuer à la consolidation de la chrono-stratigraphie du Néolithique ancien dans le Nord-Ouest de la France, et aux marges occidentales du Bassin parisien. Sur le plan méthodologique, il est donc pertinent de tenter d'exploiter au maximum un contexte relativement indigent, mais très fréquent dans le cadre préventif. Une fosse isolée découverte lors d'un diagnostic ou d'une fouille préventive est un cas fréquent. Plus localement, il nous appartiendra de discuter la nature de ce site, en particulier s'il s'agit d'une petite partie d'un site probablement plus étendu, et des critères d'implantation de celui-ci, en particulier en raison de sa localisation à une distance relativement importante des vallées et des cours d'eau $(5 \mathrm{~km})$.

\section{Présentation générale}

\section{Description de la fosse}

Il s'agit d'une fosse oblongue au contour irrégulier orientée est-ouest (fig. 2). Elle mesure un peu plus de 4,40 mètres de longueur pour une largeur maximale de 2,15 mètres. Cette dernière apparaît à une profondeur d'environ 0,5 mètre du sol actuel. Elle a été creusée dans le loss. Cette fosse est constituée de deux lobes plus profonds. Le premier couvre le tiers est de la fosse, il a une forme ovoïde et est conservé sur 0,45 mètre de profondeur. Le second, excavé au nord de la moitié ouest, a une forme irrégulière. Son fond est situé sous le niveau de décapage à 0,34 mètre de profondeur. La partie sud-ouest de la fosse est peu profonde $(0,14 \mathrm{~m})$ et a un fond relativement plat.

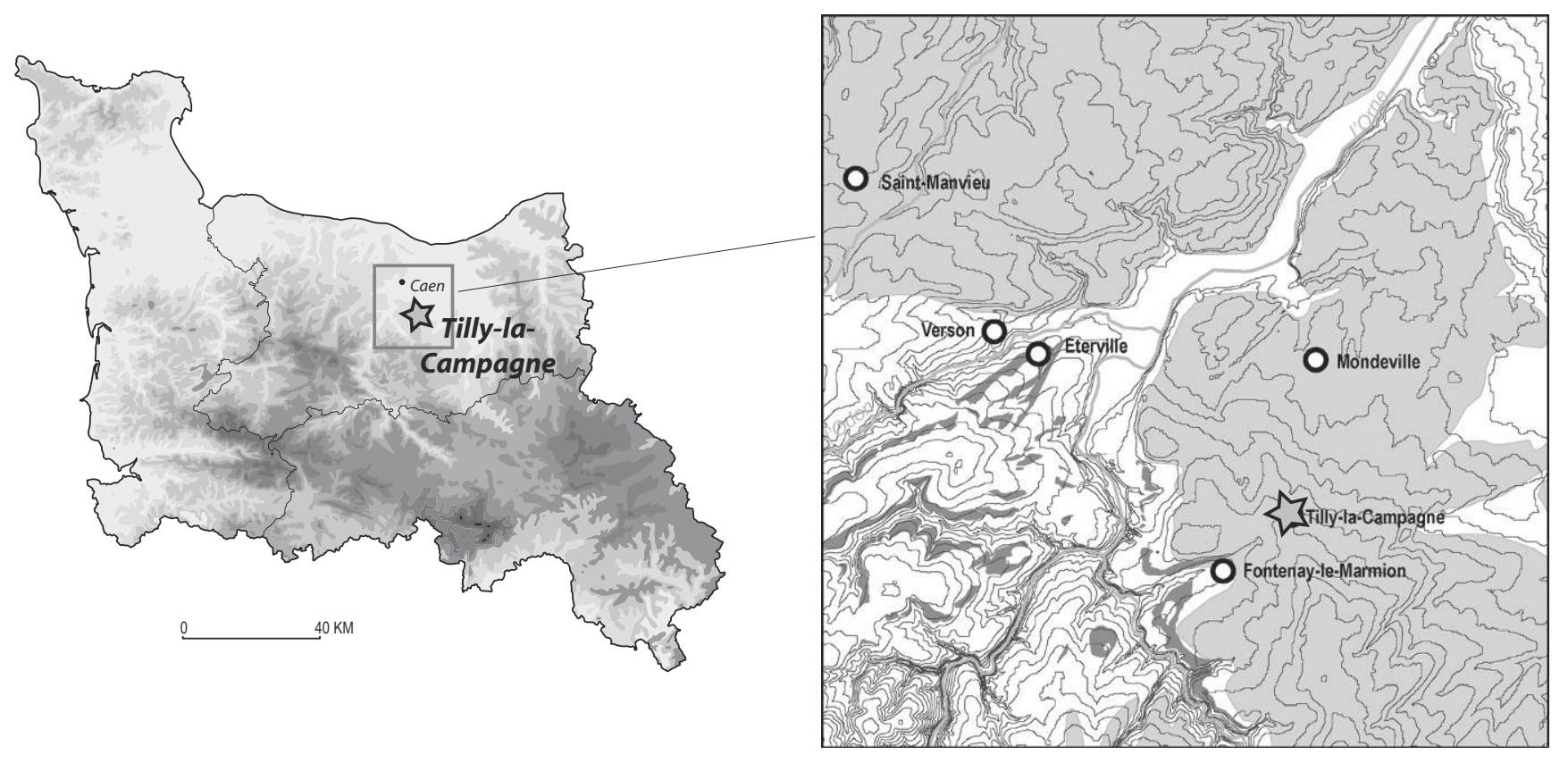

Figure 1 : Localisation du site de Tilly-la-Campagne et contexte topographique. DAO F. Charraud.

Figure 1: Location of the Tilly-la-Campagne site and its topographical context. 


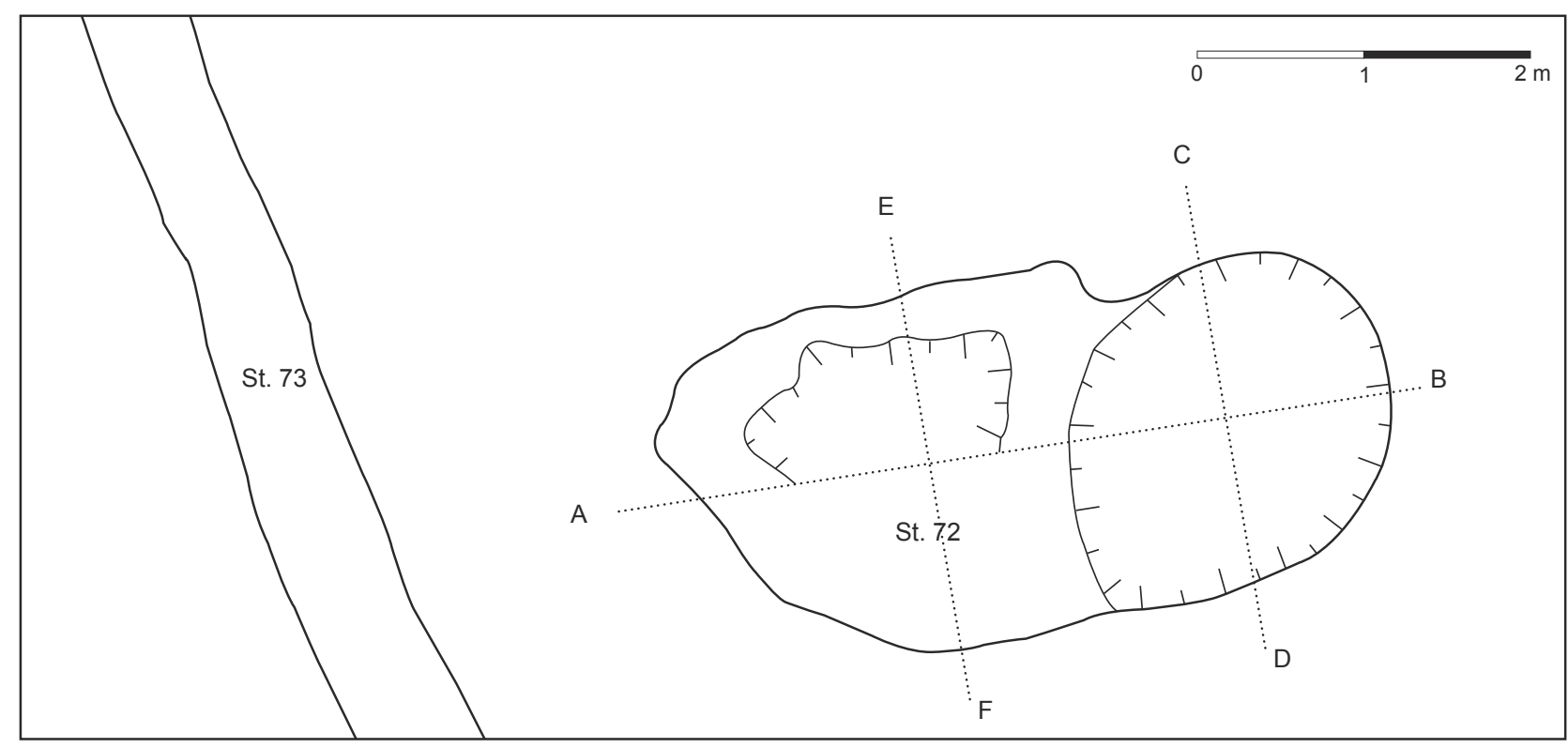

\section{A}

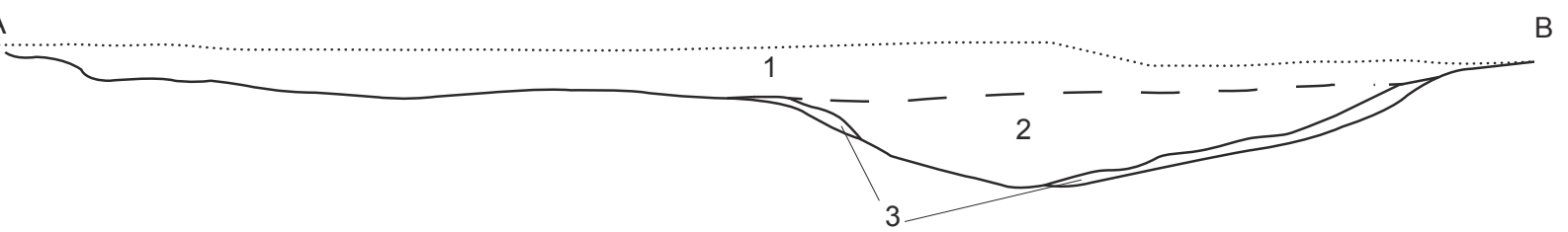

C

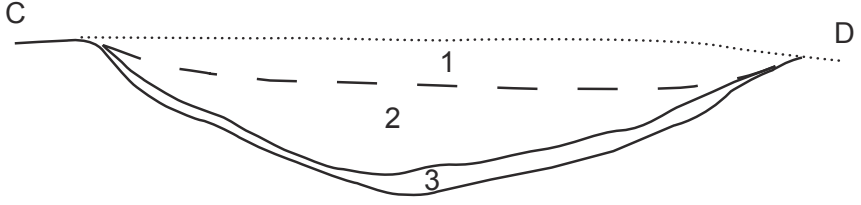

E

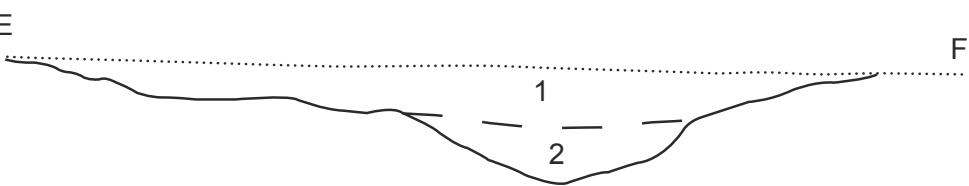

1 argilo-limoneux gris brun, charbons de bois

2 argilo-limoneux brun, organique, charbons de boi

3 loess et limon orangé

$\mathrm{F}$

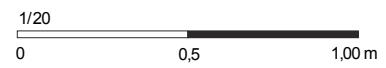

Figure 2 : Plan de la fenêtre de fouille et coupes de la structure 72. DAO P. Giraud.

Figure 2: Plan of the excavation and sections of the structure 72.

Trois remplissages ont été identifiés lors de la fouille. Le long des parois et sur le fond du lobe est de la fosse, on trouve, par endroits, une fine couche mêlant loess et limon orangé $(\mathrm{Bt})$. Elle est probablement issue de l'érosion des parois. Les parties profondes, principalement du lobe est, sont comblées par un niveau brun argilo-limoneux assez organique et charbonneux. Ce niveau est surmonté d'une couche argilo-limoneuse grise peu organique et assez charbonneuse. Cette dernière unité stratigraphique est dégradée par de nombreuses bioturbations.

L'étude micro-morphologique du comblement de la fosse, réalisée par Cécile Germain-Vallée, a permis d'observer la présence d'épais revêtements argileux hyalins dans la porosité des sédiments (Germain-Vallée et Lespez, 2011). Ces traits, très développés, témoignent d'une pédogénèse postnéolithique importante, empêchant la caractérisation de la mise en place des sédiments dans cette fosse. Ils suggèrent, par ailleurs, la reprise d'une pédogénèse lessivante sous un couvert végétal à nouveau protecteur, probablement forestier, après l'occupation du Néolithique ancien.

Létude des lames minces du comblement montre aussi que la nature des deux niveaux supérieurs est très proche. Il s'agit probablement d'un même remplissage, plus riche en matière organique vers la base. Il est envisageable que ce 
comblement corresponde à un remblai volontaire et rapide. C'est ce que tend à montrer la présence de fragments de mêmes individus céramiques répartis du sommet à la base du remplissage de la fosse.

\section{Nature et répartition du mobilier}

Le mobilier mis au jour dans la fosse 72 n'est pas abondant mais il est varié. Il a les caractéristiques des rejets domestiques que l'on trouve dans les structures accolées aux maisons de la culture Blicquy/Villeneuve-Saint-Germain (B-VSG). Il comprend du mobilier en silex (587 pièces), des fragments de poteries (199 tessons), des fragments d'anneaux en schiste (4 parures), des restes fauniques (229 restes) et des coquilles de noisettes carbonisées (aucune autre espèce végétale n'a été identifiée par l'étude carpologique de Véronique ZechMatterne). Une datation radio-isotopique a été tentée sur ces dernières, sans livrer de résultat exploitable.

Une particularité de Tilly-la-Campagne tient à la présence de onze morceaux d'outils de mouture : ils sont en calcaire coquillé ( 6 éléments), en grès ( 3 éléments) et en granite (2 éléments). Cependant, aucune surface utile n’a été mise en évidence sur ces éléments, qui ont subi diverses altérations (détermination X. Savary).

Il en va de même pour cinq nodules d'hématite oolithique d'un poids total de 5 grammes, tous de provenance locale (détermination X. Savary), également trop altérés pour permettre une quelconque lecture technique.

Près des deux tiers du mobilier a été mis au jour dans la partie est de la fosse. Il est réparti de façon relativement homogène dans les différentes passes des deux principaux niveaux du comblement. Le remplissage inférieur de la structure est pratiquement stérile.

\section{La CÉramiQue}

\section{Étude quantitative et qualitative du mobilier céramique}

Le mobilier céramique provenant de la fosse 72 est relativement pauvre puisque seulement 199 tessons (pour un poids de 226,6 g) ont été mis au jour (tableau 1). L'état de conservation général de ce mobilier est médiocre, les tessons sont très fragmentés $(1,1 \mathrm{~g} /$ tesson $)$ et assez altérés.

Bien qu'aucun élément de bord ou de préhension ne soit présent dans ce lot, huit individus sont déterminés à partir des décors et des pâtes (tableau 1). Les observations à l'œil nu et à la loupe binoculaire ont permis de déterminer deux principaux types de pâtes (tableau 2). Le premier type réunit des pâtes denses renfermant des inclusions de quartz, de fines paillettes de micas, de rares vacuoles et des nodules noirs indéterminés. Le deuxième type regroupe des pâtes poreuses et légères ne contenant aucune inclusion visible à l'œil nu.

Quatre récipients sont fins ( 5 à $7 \mathrm{~mm}$ ), deux autres contenants sont très fins $(3 \mathrm{~mm})$ et les deux derniers vases sont épais (8 à $9 \mathrm{~mm}$ ). La fragmentation des céramiques entrave l'observation des stigmates de montage. La majorité des vases semblent avoir été lissés finement. Deux individus ont des surfaces externes bosselées, très vraisemblablement imputables à un lissage grossier. Un vase est pourvu de surfaces externes et internes craquelées qui pourraient être la conséquence d'un lissage à la main mouillée. Les couleurs des surfaces externes et internes sont dans les teintes du brun-beige au brun-noir et les cœurs sont gris à noirs. Deux fragments de panse sont décorés selon la technique de l'impression. Ces impressions sont exclusivement réalisées à l'aide de peignes. Le premier individu est décoré sur trois tessons d'impressions pivotantes au peigne à deux dents, réalisées avec un outil d'une largeur de $3 \mathrm{~mm}$ (fig. $3, \mathrm{n}^{\circ} 1$ ).

\begin{tabular}{|c|c|c|c|c|c|c|c|c|c|c|c|c|}
\hline Individu & N.R. & P.N.R. (g.) & Bord & Décor & Préhens. & Etat général & Relief & Aspect & Ep. moy. & Surf. Ext. & Cœur & Surf. Int. \\
\hline $\mathrm{A}$ & 2 & 5,4 & - & - & - & peu altéré & lisse & mat & $9 \mathrm{~mm}$ & brun orangé & gris & gris brun \\
\hline B & 123 & 97,4 & - & - & - & altéré & lisse-bosselé & mat & $7 \mathrm{~mm}$ & brun beige & gris brun & gris brun \\
\hline $\mathrm{C}$ & 15 & 32,1 & - & - & - & peu altéré & lisse & mat & $7 \mathrm{~mm}$ & brun beige & gris noir & gris noir \\
\hline $\mathrm{D}$ & 1 & 0,6 & - & - & - & peu altéré & lisse-craquelé & mat & $3 \mathrm{~mm}$ & brun noir & beige & brun noir \\
\hline$E$ & 13 & 6 & - & - & - & altéré & lisse & mat & - & orangée & orangée & orangée \\
\hline $\mathrm{F}$ & 2 & 0,3 & - & - & - & peu altéré & lisse & mat & $3 \mathrm{~mm}$ & beige orangé & noir & beige orangé \\
\hline $\mathrm{G}$ & 5 & 7,1 & - & 1 & - & peu altéré & lisse & mat & $5 \mathrm{~mm}$ & brun & noir & brun \\
\hline $\mathrm{H}$ & 37 & 51 & - & - & - & peu altéré & lisse-bosselé & mat & $8 \mathrm{~mm}$ & brun beige & gris brun & gris brun \\
\hline I & 1 & 26,7 & - & 1 & - & peu altéré & lisse & mat & $6 \mathrm{~mm}$ & brun beige & noir & noir \\
\hline
\end{tabular}

Tableau 1: Données quantitatives et pondérales du mobilier de la fosse st.72.

Table 1: Quantitative and ponderal data of finds from pit 72. 


\begin{tabular}{|c|c|c|c|c|}
\hline Individu & Inclusion macroscopique & Echantillon & Inclusion microscopique & Type de pâte \\
\hline A & $\begin{array}{l}\text { paillette de micas, peu vacuoles, nodules } \\
\text { noirs }\end{array}$ & - & e & - \\
\hline B & rares inclusions, nbseuses vacuoles & $564.5 / 564.6$ & $\begin{array}{c}\text { Bioclastes fossiles micritiques/Très nbx quartz taille limon sub-sphérique sub-arrondis/Vacuoles allongées/Peu } \\
\text { vacuoles rectilignes avec élmts carbonisés/Revêtement argileux/Cristaux de calcite sparitiqur }\end{array}$ & Pâte à bioclastes fossiles \\
\hline $\mathrm{C}$ & $\begin{array}{l}\text { paillette de micas, quartz et feldpaths, } \\
\text { nodules noirs }\end{array}$ & 564.4 & $\begin{array}{c}\text { Rare gros quartz/Nbx quartz sub-arrondis sub-anguleux/Qq micas (biotite)/Ponctuation de grains } \\
\text { ferrugineux/Revêtement argileux/Vacuoles allongées }\end{array}$ & Pâte à particules sableuses \\
\hline $\mathrm{D}$ & $\begin{array}{l}\text { paillette de micas, } 1 \text { élément de roche, } \\
\text { nodules noirs }\end{array}$ & - & 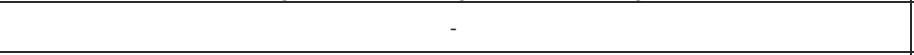 & - \\
\hline $\mathrm{E}$ & vacuoles & - & - & - \\
\hline $\mathrm{F}$ & - & - & - & - \\
\hline G & paillette de micas, quartz & 564.2 & $\begin{array}{c}\text { Peu quartz anguleux et allongés sable grossier/Nbx quartz sub-arrondis sables fins et limons/1 oolithe } \\
\text { ferrugineuse/Chamotte? }\end{array}$ & Pâte à particules sableuses \\
\hline $\mathrm{H}$ & rares inclusions, vacuoles & 564.3 & $\begin{array}{l}\text { Bioclastes fossiles micritiques/Très nbx quartz taille limon sub-sphérique sub-arrondis/Vacuoles allongées/Très } \\
\text { rare élmts carbonisés dans vacuoles/Revêtement argileux }\end{array}$ & Pâte à bioclastes fossiles \\
\hline I & $\begin{array}{l}\text { quartz, feldpaths, paillette de micas, } \\
\text { schistes? nodules ferrugineux }\end{array}$ & 564.1 & $\begin{array}{c}\text { Bcp quartz sub-anguleux et quartz poly engrenés/Rares feldspaths/Micas (biotite et } 1 \text { petite muscovite)/Qq } \\
\text { bioclastes fossiles }\end{array}$ & Pâte à particules sableuses \\
\hline
\end{tabular}

Tableau 2 : Données descriptives des pâtes des céramiques de la fosse st.72.

Table 2: Description of the pottery types from pit 72.

La thématique du décor est indéterminée. Cependant, au moins quatre bandes verticales d'impressions au peigne sont observées. Les impressions paraissent dévier à la base d'une bande, mieux conservée, pour former potentiellement un décor de guirlande. Le second individu est décoré d'impressions pivotantes au peigne à quatre dents. L'outil employé dans ce décor est large de $6 \mathrm{~mm}$ avec deux dents très mal individualisées (fig. 3, $\mathrm{n}^{\circ}$ 2). La thématique décorative est mal perçue d'autant qu'une partie des impressions a disparu. Néanmoins, il est possible d'observer au moins huit bandes d'impression verticales, qui composent vraisemblablement un décor couvrant.

\section{Étude pétrographique des pâtes céramiques}

Dans un premier temps, l'observation à l'œil nu et à la loupe binoculaire du mobilier céramique a permis d'identifier deux principaux groupes de pâtes céramiques. Il s'agit de pâtes à particules sableuses et de pâtes vacuolaires. Une analyse microscopique des pâtes a été réalisée afin de confirmer et de préciser le contenu des groupes macroscopiques. Ce travail s'est également porté sur la possible présence de dégraissant végétal dans le cadre d'un travail de recherche universitaire (Jan, 2010 et 2011). De ce fait, six échantillons ont été prélevés sur le mobilier céramique pour être étudiés au microscope polarisant (tab. 2).

Une première catégorie regroupe les pâtes à bioclastes fossiles $(564.3,564.5,564.6)$. Les principaux éléments figurés sont des organismes fossiles à squelette calcaire. Ce sont pour les plus reconnaissables des fragments d'échinodermes, de bryozoaires, de foraminifères et de mollusques (lamellibranches et gastéropodes). Ces bioclastes sont plutôt de petites dimensions (entre 200 et $400 \mu \mathrm{m}$, avec de nombreux fragments inférieurs à $100 \mu \mathrm{m}$ ). Ils sont parfois accompagnés de rares éléments plus grossiers de l'ordre du millimètre. L'échantillon 564.3 contient légèrement plus de

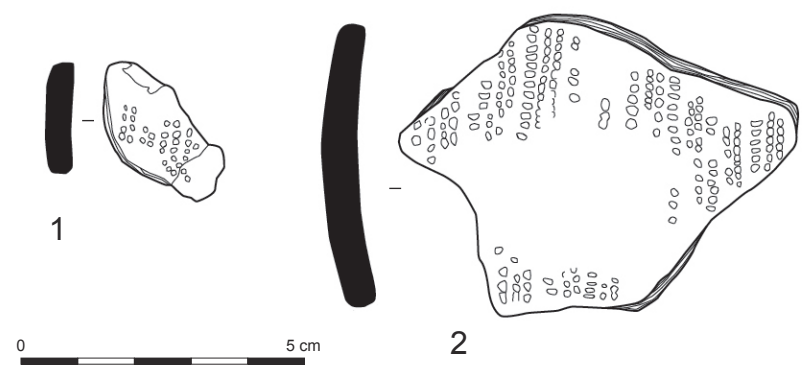

Figure 3 : Céramiques décorées. Dessins D. Jan. Figure 3: Decorated pottery.

bioclastes pluri-millimétriques. Dans ces pâtes, les bioclastes sont seulement visibles en lumière naturelle sous la forme de "fantômes ». Ces éléments ont disparu soit lors de la décarbonatation des limons d'origine, soit par une forte cuisson des vases et de l'enfouissement de ces derniers. Les quelques vacuoles millimétriques rencontrées dans ces pâtes correspondent très certainement à la disparition complète des bioclastes induisant cet aspect très vacuolaire en macroscopie. Ces premiers éléments sont accompagnés de nombreux quartzs sub-sphériques et sub-arrondis de la taille des limons grossiers. Des revêtements argileux sont aussi observés près des surfaces pour ces trois échantillons. Quelques éléments carbonisés sont présents dans certaines vacuoles de l'échantillon 564.3 mais la rareté de ces inclusions réfute l'emploi d'un dégraissant végétal. Les deux autres échantillons comportent des vides rectilignes de 3 à $5 \mathrm{~mm}$ de long pour une largeur d'environ $200 \mu \mathrm{m}$. Ces éléments renferment un filament carbonisé induisant la disparition de matière organique. Cette matière végétale pourrait correspondre à des restes de tiges de plantes à fleur du type poacées. Cependant, la rareté de ces végétaux dans les échantillons est en contradiction avec un emploi comme dégraissant. Une pâte assez proche de ces dernières est utilisée pour réaliser le vase à 
décor de cordons en V du site de Mondeville " le Haut Saint-Martin » (Chancerel et al., 2006).

Une seconde catégorie regroupe les pâtes à particules sableuses $(564.1,564.2,564.4)$. Deux échantillons renferment une quantité importante de grains de quartz monocristallins sub-sphériques et sub-anguleux de la taille des limons grossiers. Ces derniers sont accompagnés dans l'échantillon 564.1 de rares feldspaths (orthoses), quelques micas (biotites et muscovite) et accessoirement de rares bioclastes fossiles. Ils sont associés dans l'échantillon 564.4 à quelques micas (biotites), à des ponctuations de grains ferrugineux et à des vacuoles dont la forme rappelle sensiblement celle de coquille de bioclastes. Des revêtements argileux sont identifiés dans cette pâte. Le dernier échantillon se démarque des deux autres par la présence de sables grossiers quartzeux allongés et anguleux tandis que les sables fins et limon grossiers sont moins présents. Cet échantillon renferme des grains dont la couleur contraste avec le reste de la pâte. Ces grains pourraient être interprétés comme de la chamotte ou plus vraisemblablement comme des grumeaux d'argiles issus d'un mauvais malaxage ou d'une évolution pédo-génétique puisque les minéraux qu'ils contiennent ne se démarquent pas de ceux rencontrés dans la pâte. Les pâtes céramiques de Fontenay-le-Marmion « le Grand Champ » et de Mondeville " le Haut Saint-Martin » comportent également des particules sableuses (Chancerel et al., 2006; Giraud et al., 2012).

Cette étude pétrographique, menée sur une partie importante des individus céramiques identifiés, confirme les groupes macroscopiques de pâtes. Une partie des récipients sont confectionnés à partir d'argile renfermant des particules sableuses. L'absence d'éléments discriminants dans ces dernières exclut toute détermination de provenance. Les autres vases sont réalisés avec des limons ou marnes bioclastiques omniprésents dans la Plaine de Caen. Ces éléments fossiles calcaires se sont détériorés durant la cuisson et l'enfouissement des tessons, ce qui entraine l'aspect vacuolaire de ce groupe de pâte. Dans cette fosse, on remarque l'absence de céramique confectionnée à partir d'argile issue de l'altération d'une roche magmatique alors qu'elles sont bien observées sur les sites de Fontenay-le-Marmion et de Mondeville (Chancerel et al., 2006; Giraud et al., 2012).

\section{Conclusion de l'étude du mobilier céramique}

Ce lot apporte quelques nouveaux éléments sur le début du Néolithique dans le Calvados. Les éléments remarquables de ce corpus consistent en deux décors d'impressions au peigne. Cette technique décorative bien qu'utilisée pour des périodes plus récentes (protohistoire) reste néanmoins significative des groupes culturels de tradition danubienne. En tenant compte de l'ensemble du mobilier, ce lot céra- mique s'inscrit dans la culture B-VSG. Cependant, il semble utopique de rattacher ce corpus à une phase particulière de cette culture de par la faiblesse des éléments caractéristiques. L'infime quantité de céramiques conservées est comparable à certains sites B-VSG mis au jour localement. En effet, 125 tessons ont été ramassé dans les fosses de la maison de Mondeville " le Haut Saint-Martin » et 261 fragments de céramique $(1,094 \mathrm{~kg})$ proviennent de celles de la maison de Fontenay-le-Marmion «le Grand Champ » (Chancerel et al., 2006; Giraud et al., 2012). La faiblesse des corpus est peut-être à mettre en relation avec l'arasement des structures anciennes imputable à la mise en culture considérable des terres de la campagne de Caen. La forte fragmentation et l'altération observée sur le matériel montrent que ces débris de vases sont issus de rejets secondaires. Aucune forme céramique bien conservée n’a été mise au jour ce qui pourrait être un argument supplémentaire pour sous-entendre qu'il ne s'agit pas de rejet direct dans la fosse. De plus, la dissémination de certains récipients, de la surface à la base de la fosse, semble montrer que le comblement de cette structure a été assez rapide.

\section{LA FAUNE}

Un petit ensemble de 229 restes osseux pour un poids total de $269 \mathrm{~g}$. provient de la structure 72 . Ces ossements sont toutefois fortement abîmés et fragmentés, ce qui a rendu extrêmement difficile les phases d'identification et d'observation. Seule une brève présentation en termes de présence et d'absence des espèces et des parties anatomiques peut être réalisée. Il est toutefois important de noter que les données archéozoologiques, dans cette région et pour cette période chronologique, sont encore exceptionnelles. Il est, par conséquent, apparu intéressant de mentionner l'existence de ce petit lot.

Ce sont les restes de bovins qui sont les plus nombreux avec vingt-quatre ossements décomptés pour un poids de 159 g. Ces restes appartiennent à des esquilles d'os longs ou bien à des fragments de crânes et de dents. Ils n'ont malheureusement pu être déterminés au rang de l'espèce (bœuf ou aurochs) étant donné la forte détérioration de la surface osseuse et l'importante fragmentation. Pour les restes d'ovi-caprinés, la différenciation mouton/chèvre n'a pu être réalisée, et les suidés sont également présents mais dans de moindres proportions avec respectivement quatorze et neuf restes déterminés. La part des petits mammifères domestiques semble sous-évaluée et est vraisemblablement à attribuer au phénomène de conservation différentielle, tout comme l'absence de taxons sauvages. Un ensemble de 182 restes, non identifiés aux rangs taxinomique et anato- 
mique, complète le lot. Ce sont majoritairement des fragments d'os brûlés (os carbonisés $\mathrm{n}=11$; os calcinés $\mathrm{n}=$ 86) dont le contact avec le feu a entraîné une importante fragmentation et la suppression des critères de détermination. Il est intéressant de noter que les os calcinés paraissent détenir un plus fort potentiel de conservation face au $\mathrm{pH}$ acide du sol. Cet élément, observé notamment sur plusieurs ensembles archéozoologiques bretons, semble s'expliquer par le fait que la calcination détruit la matière organique. Les os brûlés, à de très fortes températures, deviennent alors moins sujets à la dissolution (Baudry, 2012).

L'impact de la conservation différentielle se traduit également au sein de la distribution anatomique. Cet ensemble est ainsi majoritairement composé de fragments de tête avec la présence de mandibules $(\mathrm{n}=8)$ et de dents isolées $(\mathrm{n}=$ 24). Les vertèbres $(n=1)$, les côtes $(n=9)$, les ceintures $(n=$ $1)$, les os longs $(n=3)$ et les bas-de-patte $(n=4)$ s'illustrent plus faiblement. La répartition des restes montre ainsi des déséquilibres entre les différentes parties du squelette et ceci quelle que soit l'espèce prise en compte. Malgré la faiblesse numérique de certains éléments, la présence de l'ensemble des parties du squelette est toutefois importante à noter car elle suggère que ces espèces ont été présentes sous la forme de bêtes sur pieds et non seulement de quartiers de viande.

\section{LE MOBILIER EN SCHISTE}

Cinq éléments en schiste ont été mis au jour dans la fosse 72. À l'exception d'un seul (fig. 4, no 3), tous ont été trouvés dans la partie est de la structure. Le premier est un fragment de plaque de forme rectangulaire, découvert en surface de la fosse (fig. $4, \mathrm{n}^{\circ} 1$ ). Il mesure $3 \mathrm{~cm}$ de longueur pour $2,5 \mathrm{~cm}$ de largeur et $0,6 \mathrm{~cm}$ d'épaisseur. Il pourrait s'agir d'un rebut de fabrication d'un bracelet.

L'objet $\mathrm{n}^{\circ} 2$ correspond à un anneau irrégulier probablement dégradé par le feu (fig. 4, $\mathrm{n}^{\circ} 2$ ). La largeur maximale de l'anneau mesure $2,2 \mathrm{~cm}$ et la minimale $1,6 \mathrm{~cm}$; son épaisseur est de $0,7 \mathrm{~cm}$. Le diamètre interne est compris entre 5 et $7 \mathrm{~cm}$. Sa mise en forme semble inachevée. Nous pouvons supposer que cet objet est une ébauche de bracelet.

L'anneau $\mathrm{n}^{\circ} 3$, trouvé à moins de $5 \mathrm{~cm}$ de profondeur dans la portion nord-ouest de la fosse, est façonné dans du schiste tacheté (fig. 4, no 3). Il a la particularité d'avoir une extrémité externe saillante. Ce fragment est de petite dimension avec une largeur de $1,4 \mathrm{~cm}$ et une épaisseur de $0,5 \mathrm{~cm}$. Le diamètre interne de l'anneau mesure entre 6 et $7 \mathrm{~cm}$.

L'objet $\mathrm{n}^{\circ} 4$ est un fragment de bracelet à très large couronne en schiste tacheté (fig. $4, \mathrm{n}^{\circ}$ 4). L'anneau mesure $4 \mathrm{~cm}$ de largeur pour $0,9 \mathrm{~cm}$ d'épaisseur. Le diamètre interne est estimé à environ $7 \mathrm{~cm}$.
Lobjet $\mathrm{n}^{\circ} 5$ est un fragment d'anneau en schiste tacheté de 6 à $7 \mathrm{~cm}$ de diamètre (fig. 4, $\mathrm{n}^{\circ}$ 5). Il mesure $1,4 \mathrm{~cm}$ de largeur pour $0,5 \mathrm{~cm}$ d'épaisseur.

Le dernier élément est un demi-anneau en schiste du Pissot (Orne, Fromont, 2013). Il provient de la base du comblement principal de la fosse. La largeur de la couronne mesure $1,85 \mathrm{~cm}$ pour $1 \mathrm{~cm}$ d'épaisseur et un diamètre de $6,5 \mathrm{~cm}$ (fig. $4, \mathrm{n}^{\circ}$ ).

Les bracelets à large couronne sont assez rares dans les contextes régionaux des phases ancienne et moyenne du Villeneuve-Saint-Germain; aucun bracelet fini, dont la largeur de l'anneau dépasse $3,5 \mathrm{~cm}$, n'en provient. Il semble qu'en Normandie, comme dans le Bassin parisien, la largeur des couronnes des bracelets en schiste a tendance à s'élargir à partir de la phase moyenne du Villeneuve-Saint-Germain. La présence, dans ce lot restreint, d'un fragment de bracelet à anneau plat et couronne très large est peut-être un indice pour une attribution de ce contexte à la dernière phase du B-VSG.

\section{LE MOBILIER EN SILEX}

Tout le mobilier étudié provient de la structure 72. Il a été pris en compte comme un ensemble homogène, car aucun indice ne remet en question son unité. Le contexte de découverte et la nature du mobilier n’ont pas justifié la réalisation d'une étude fonctionnelle. L'étude de l'outillage est donc principalement réalisée à partir de critères typologiques, morphologiques et techniques généraux.

La méthode globale de l'étude est celle que nous avons expérimentée sur la plupart des ensembles du Néolithique ancien de Basse-Normandie (Charraud, 2013).

La structure 72 comprend un total de 587 vestiges en silex, parmi lesquels on compte près de deux tiers de débris (374 débris, soit $64 \%$; tabl. 3). Les déchets de taille techniquement identifiables concernent 135 objets (23\%), ils renvoient à un débitage d'éclats multidirectionnel côtoyant un débitage laminaire standardisé réalisé par percussion indirecte. L'outillage comprend 78 objets (13\%). Une part d'outils façonnés, correspondant à une norme typologique, est complétée par un outillage expédient, qui ne correspond à aucun schéma prédéfini.

\section{LA MATIÈRE PREMIÈRE, ORIGINE ET ÉCONOMIE}

À l'exception d'un fragment mésial de lame en silex indéterminé, le seul matériau reconnu est le silex du Cinglais. Ce matériau a fait l'objet d'une caractérisation pétrographique de $S$. Coutard d'après des observations en lames minces 

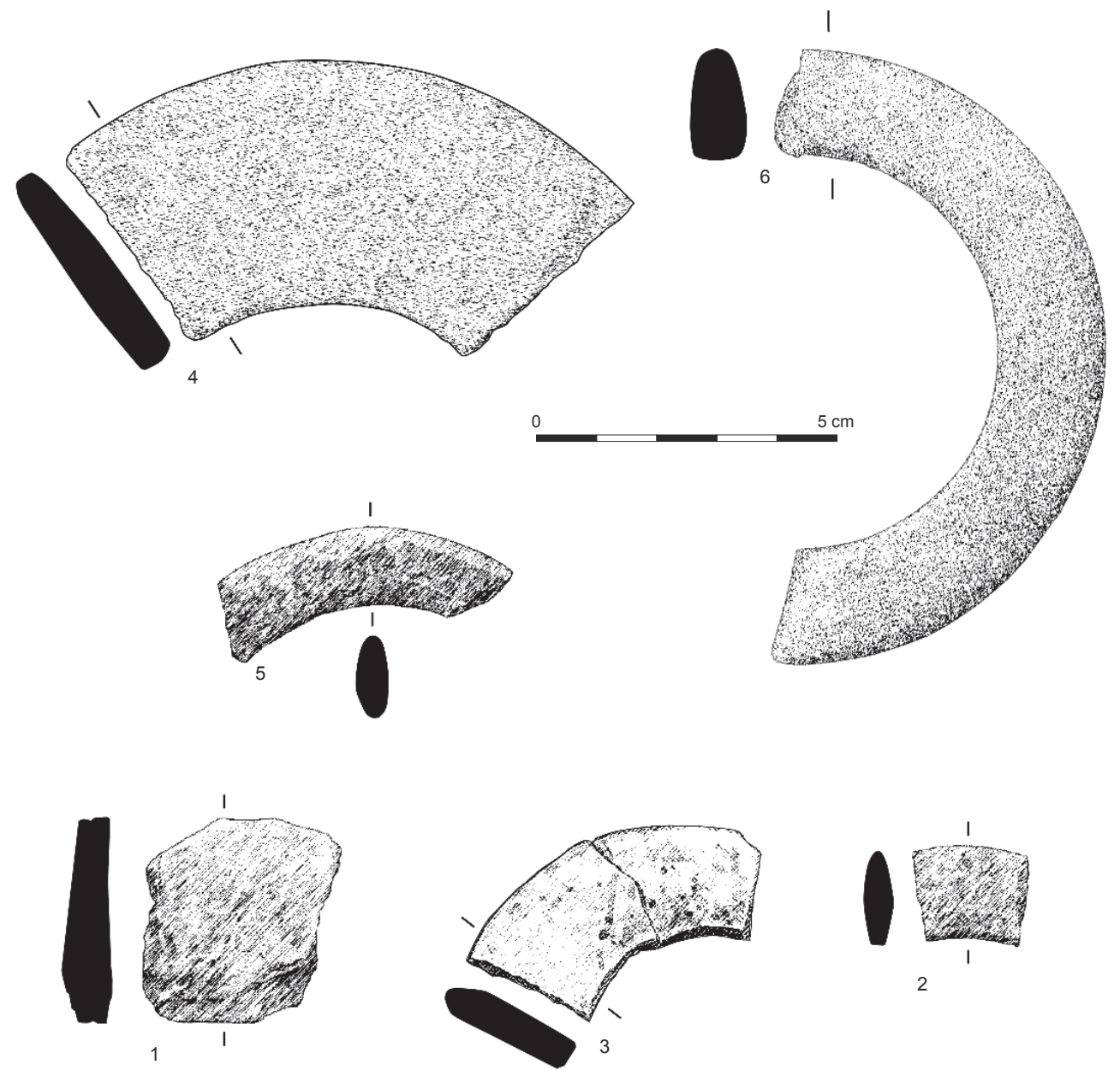

Figure 4: Mobilier en schiste. Dessins A. Cocollos.

Figure 4: Schist objects.

(Coutard, 1998). C'est un silex d'aspect " marron glacé ", à grain fin et à cortex d'épaisseur variable (de 4 à $25 \mathrm{~mm}$ ) à zone sous-corticale blanchâtre à limite floue, qui se présente sous la forme de rognons de 20 à $30 \mathrm{~cm}$ à l'affleurement. Il s'agit d'une calcédonite cryptocristalline à nombreux pelloïdes, comportant dans certains cas des bioclastes de grande taille (> $300 \mu \mathrm{m})$, et une microfaune composée de nombreux spicules et entroques. Il comporte en outre des oxydes de fer (goethite), occasionnellement organisés en amas (en zone sous-corticale) et présente parfois un litage concentrique.

Ce silex est présent sur la plupart des sites du Néolithique ancien du Calvados pour le débitage de lames standardisées par percussion indirecte (Charraud, 2013). Les deux sources reconnues du silex du Cinglais se trouvent sur le plateau du Cinglais et dans la zone de Soumont-Saint-Quentin, respectivement à $17 \mathrm{~km}$ et $20 \mathrm{~km}$ de Tilly-la-Campagne vers le sud et le sud-ouest.

La qualité des productions mises en œuvre dans ce silex, non seulement à Tilly, mais dans la plupart des sites du Néolithique ancien de Basse-Normandie, supposent un silex d'excellente qualité, uniquement disponible sur les gîtes à une profondeur minimale de deux mètres sous la surface actuelle. Sans cela, les blocs présents en surface sont toujours altérés par la gélifraction et inaptes à l'application d'un schéma opératoire complexe. Ces productions sont donc la preuve indirecte que les matériaux taillés au Néolithique ancien ont été acquis au moyen de structures d'extraction comparables à celles mises en évidence à Espins "Foupendant " (Charraud, 2015) et à Soumont-SaintQuentin "les Longrais" (Ghesquière et al., 2008).

La composition des vestiges en silex du Cinglais à Tilly est comparable à celle des autres habitats du Néolithique ancien des plaines jurassiques bas-normandes.

Les témoins des premières phases d'exploitation des rognons (dégrossissage, mise en forme) sont relativement rares et concernent principalement l'ablation de plages corticales résiduelles. Il ne s'agit pas d'éclats de dégrossissage massifs comme on en trouve sur les gites. La proportion de $20 \%$ de tels déchets correspond à ce qui a été reconnu à Fontenay-le-Marmion « le Grand Champ " (Giraud et al., 2012). Ces données rendent compte d'un fractionnement spatial de la chaîne opératoire laminaire en silex du Cinglais 
Déchets de taille

\begin{tabular}{|l|c|}
\hline Eclat cortical brut & 21 \\
\hline Eclat de dégrossissage épais (é.> 3 cm) & 1 \\
\hline Lame corticale (>50 \%) & 5 \\
\hline Débris, casson cortical & 3 \\
\hline Eclat brut court & 26 \\
\hline Eclat brut allongé & 6 \\
\hline NE multidirectionnel & 1 \\
\hline Fragment/casson N.E. & 2 \\
\hline Eclat de préforme NL & 4 \\
\hline Eclat de préparation de crête & 4 \\
\hline Lame à crête & 2 \\
\hline Lame sous crête & 4 \\
\hline Tablette d'avivage & 1 \\
\hline Eclat entretien PDF & 2 \\
\hline Entretien flanc N.L. longitudinal & 5 \\
\hline Entretien flanc N.L. transversal & 1 \\
\hline Lame technique/lame d'entretien N.L. & 3 \\
\hline Nucléus à lames & 1 \\
\hline Fragment/casson N.L. & 2 \\
\hline Lame entière percu indirecte & 2 \\
\hline Lame fragment prox. percu directe & \\
\hline Lame fragment prox. percu indirecte & 13 \\
\hline Lame fragment prox. percu indéterminée & \\
\hline Lame fragment mésial & 10 \\
\hline Lame fragment distal & 5 \\
\hline Lamelle ou fragment & 3 \\
\hline Chute de burin & 6 \\
\hline Ebauche indifférenciée ou bifaciale & 2 \\
\hline TOTAL DECHETS DE TAlLLE & \\
\hline
\end{tabular}

Débris et indéterminés

\begin{tabular}{|l|c|}
\hline Esquille & 349 \\
\hline Casson d'éclat & 24 \\
\hline Autre, débris thermique & 1 \\
\hline TOTAL DEBRIS & 374 \\
\hline
\end{tabular}

\section{Outils}

\begin{tabular}{|l|c|}
\hline Tranchet bifacial & 1 \\
\hline Fragment pièce bouchardée & 14 \\
\hline Mèche & 1 \\
\hline Grattoir sur lame & 1 \\
\hline Burin sur lame & 5 \\
\hline Lame tronquée & 2 \\
\hline Perçoir & 1 \\
\hline Eclat épais retouché & 10 \\
\hline Eclat épais denticulé & 4 \\
\hline Eclat épais écaillé/esquillé & 6 \\
\hline Eclat mince retouché & 4 \\
\hline Eclat mince écaillé/esquillé & 4 \\
\hline Pièce esquillée & 2 \\
\hline Lame retouchée & 2 \\
\hline Lame écaillée (écaillures unifaciales) & 20 \\
\hline Lame esquillée (écaillures bifaciales) & 1 \\
\hline TOTAL OUTILLAGE & 78 \\
\hline
\end{tabular}

\section{TOTAL SERIE}

Tableau3 : Décompte du mobilier lithique.

Table 3: Total of lithic material. dans la région. Le dégrossissage des blocs est toujours effectué sur les gîtes, seul le plein débitage laminaire est effectué sur les habitats. Les étapes de la chaîne opératoire représentées sur les différents sites sont complémentaires :

- Les déchets de dégrossissage sont fortement représentés sur les gîtes et très peu dans les habitats;

- Il en va de même pour les nucléus à lames, rares en contexte d'habitat et abondants sur les mines;

- Les supports laminaires, bruts ou transformés en outils, sont rares sur les mines et abondants dans les habitats.

Ce fractionnement de la chaîne opératoire n'est pas une spécificité régionale : il a été démontré que pour de nombreux types d'industries lithiques, qu'il s'agisse d'exemples préhistoriques bien documentés ou d'exemples ethnographiques, certaines phases-clés de la chaîne opératoire s'exercent très souvent sur les gîtes de matière première ou à proximité immédiate (Pétrequin et Pétrequin, 1993; Pelegrin, 1995).

Un fragment mésial de lame est réalisé dans un silex indéterminé, en raison de la patine importante qui l'affecte (fig. 5, $\mathrm{n}^{\circ} 7$ ). Il ressemble fortement au silex tertiaire bartonien caractérisé dans le Bassin parisien (Allard et al., 2010), mais son altération empêche d'être affirmatif.

\section{Caractères technologiques DES DÉCHETS DE TAILLE}

On a observé que les blocs de silex du Cinglais parviennent déjà préparés sur le site, seul le plein débitage laminaire est effectué sur place. À partir de la phase de mise en place des crêtes antérieures, tout le débitage laminaire est représenté.

Le débitage laminaire en silex du Cinglais est comparable à ce qui a été décrit dans le Bassin parisien et la Belgique au Néolithique ancien (Bostyn, 1994; Allard, 2005), moyennant quelques différences techniques anecdotiques et un module de supports parfois moins important (Charraud, 2013).

La proportion des déchets caractéristiques du débitage laminaire est comparable aux autres sites du Néolithique ancien de la région : $21 \%$ des déchets de taille (lames et fragments non compris), contre $31 \%$ à Démouville (Charraud, 2013) ou $17 \%$ à Mondeville (Chancerel et al., 2006). La phase de préparation des crêtes comprend huit éclats caractéristiques, dont deux présentent des plages corticales résiduelles. On remarque qu'une sélection de rognons de petite taille a probablement été effectuée sur les gîtes, car la mise en forme des nucléus en vue du plein débitage laminaire débute à un stade très précoce de l'exploitation des blocs.

Aucune différence qualitative dans le débitage laminaire n'a été observée par rapport aux productions du plateau 
du Cinglais. La proportion des déchets de taille laminaire montre que les lames présentes ont probablement été débitées sur place : rien ne permet de supposer, contrairement à Mondeville (ibid.), l'apport de supports déjà débités. À l'inverse, leur nombre exclut l'idée d'une production excédentaire : on compte trente-trois lames (deux entières et trente et un fragments), auxquels s'ajoutent trente et un outils sur lame. La production des supports répond donc aux besoins fonctionnels locaux, il n'y a pas de redistribution perceptible.

Comme c'est souvent le cas dans les habitats de la Plaine de Caen, les nucléus à lame sont très rares : trois fragments sont présents dans la série. Les derniers enlèvements obtenus sont compris entre 7 et $10 \mathrm{~cm}$, ce qui correspond au module moyen constaté sur les autres assemblages régionaux (Charraud, 2013). Pour le reste, la série ne permet pas de décrire des gestes technologiques significatifs à une échelle macroéconomique.

La technique de détachement des supports laminaires est la percussion indirecte pour tous les supports déterminés; cette proportion est conforme aux autres assemblages régionaux : elle concerne souvent plus de $90 \%$ des supports, y compris pour les assemblages importants comme Verson (Germain-Vallée et al., 2014), à l'exception de Fontenay-leMarmion où elle n'est que de $70 \%$ (Giraud et al., 2012). La percussion indirecte concerne également deux éclats de mise en forme de crêtes.

Le débitage d'éclats, minoritaire au sein de l'assemblage, est représenté par au moins trente-deux éclats de plein débitage avérés, ainsi qu'un nucléus à éclats et deux fragments. Aucun schéma opératoire récurrent ne peut être mis en évidence pour ce débitage. Il s’agit d'un débitage multidirectionnel non structuré réalisé par percussion directe dure. Les supports sont clairement produits sur place pour les besoins immédiats de l'outillage. Cette production intervient en complément de la production laminaire, elle fournit un apport supplémentaire de supports destinés à l'outillage sur éclat et l'outillage expédient. En dehors de sites normands du Villeneuve-Saint-Germain dont la production d'éclats est la seule production réalisée (Écouché "Carrière MEAC » : Marcigny et al., 2010), les sites de Basse-Normandie ont souvent recours à des ressources très locales de piètre qualité pour cette production complémentaire. C'est le cas à Colombelles (Billard et al., 2014), à Verson (GermainVallée et al., 2014) et Saint-Manvieu-Norrey (Chancerel $e t$ al., 1992). En revanche, le silex du Cinglais est parfois le seul utilisé, comme à Tilly-la-Campagne, pour la production laminaire comme pour la production d'éclats : il en va de même à Fontenay-le-Marmion (Giraud et al., 2012).

\section{L'outillage}

L'outillage (78 outils) est réalisé à $40 \%$ sur lame, $40 \%$ sur éclats et $20 \%$ sur masse centrale. Malgré la faiblesse de l'effectif, ce sont des proportions très semblables à celles de Fontenay-le-Marmion (ibid.). Ici encore, la composition est très classique par rapport aux autres ensembles régionaux. De même, l'outillage ne diffère pas des autres assemblages du Néolithique ancien par sa typologie. Les outils sur lame sont dominés par les lames retouchées et esquillées (21 exemplaires : fig. $5, \mathrm{n}^{\text {os }} 5$ à 9) : ces outils résultent vraisemblablement d'utilisations expédientes de supports bruts.

Les burins sur lame (5) sont bien représentés (fig. 5, nos 5 à 9), comme dans tous les sites du Villeneuve-Saint-Germain en Normandie. Il s'agit de burins sur cassure, dont tous les supports conservent les traces d'une utilisation antérieure à l'aménagement du coup de burin. L'outillage sur lame comprend également, comme outils façonnés, un seul grattoir sur lame, (fig. $5, \mathrm{n}^{\circ}$ 4) ainsi que deux lames tronquées, dont l'utilisation comme armatures de faucille est possible, en dépit de l'absence de lustré visible à l'œil nu (fig. 5, no 9).

Quelques déchets témoignent d'activités de taille de silex (14 fragments de pièces bouchardées), ou de transformation d'outils (6 chutes de burin).

Les outils sur éclat et sur masse centrale sont essentiellement des outils expédients, à l'exception d'un tranchet réalisé sur un gros éclat (fig. $6, \mathrm{n}^{\circ} 1$ ). Ses bords sont très rectilignes. Son tranchant n'est pas déterminé par un coup de tranchet mais par une surface d'éclatement présente antérieurement à la mise en forme du support. La mise en forme de cette pièce à la morphologie plano-convexe, est réalisée au moyen d'une retouche directe couvrante, antérieure à l'amincissement de la partie basale du support par retouche inverse plate. Ces outils sont souvent considérés comme étant des caractères récents des assemblages du VilleneuveSaint-Germain (Bostyn, 1994), mais des exceptions existent.

Du reste, l'outillage sur éclat comprend un perçoir et une mèche courte. Il est uniquement composé d'outils sur éclat, retouchés (14: fig. $6, \mathrm{n}^{\circ} 2$ ), esquillés et écaillés (10) ou denticulés ( $4:$ fig. $6, n^{\circ} 3$ ). Ces outils expédients ne correspondent par définition à aucune norme typologique; il s'agit d'outils rapidement produits, correspondant généralement à des utilisations brèves et à des gestuelles simples (Bostyn et Allard, 2006; Guilbert et al., 2006). Deux pièces esquillées complètent ce panel (fig. 6, $\mathrm{n}^{\circ} 4$ ). Il s'agit d'éclats bruts ou sommairement mis en forme, probablement employés en percussion indirecte, entre un percuteur et la matière travaillée (coins?). Les exemplaires de Tilly-la-Campagne sont particulièrement usés. 

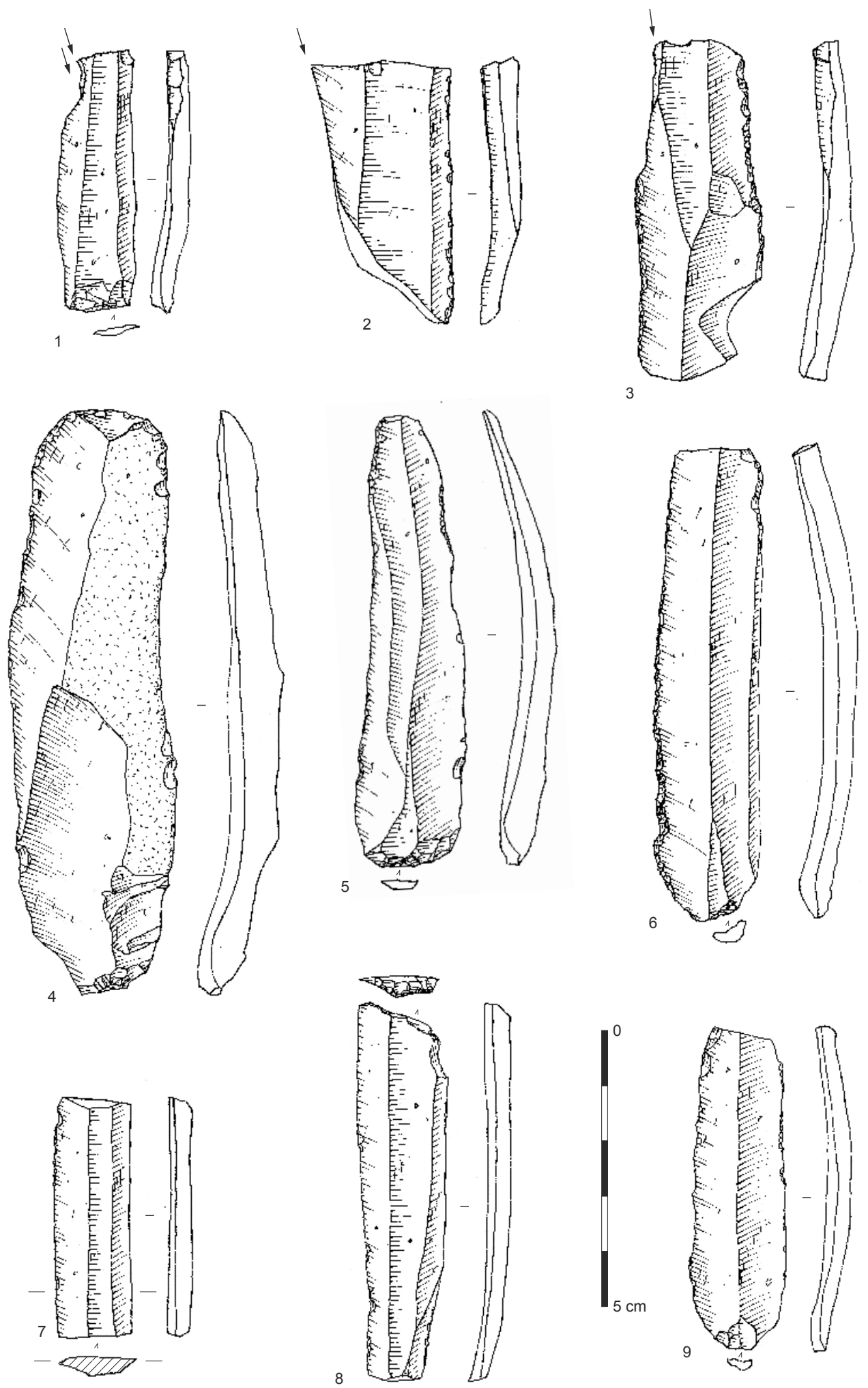

Figure 5 : Tilly-la-Campagne, structure 72 : mobilier lithique, outils sur lame. 1 à 3 : burins sur lame; 4 : grattoir sur lame; 5 à $9:$ lames écaillées/esquillées (ou $\mathrm{n}^{\circ} 7$ : fragment mésial). Le fragment mésial de lame, $\mathrm{n}^{\circ} 7$ est le seul élément en matière première indéterminée. Dessins F. Charraud.

Figure 5: Tilly-la-Campagne, structure 72: lithic material, Blade tools. 1 à 3: Blade burins; 4: Blade scraper; 5 à 9: worked blades (ou nº: mesial fragment). Le mesial blade fragment $n^{\circ} 7$ is the only element where the raw material has not been determined. 

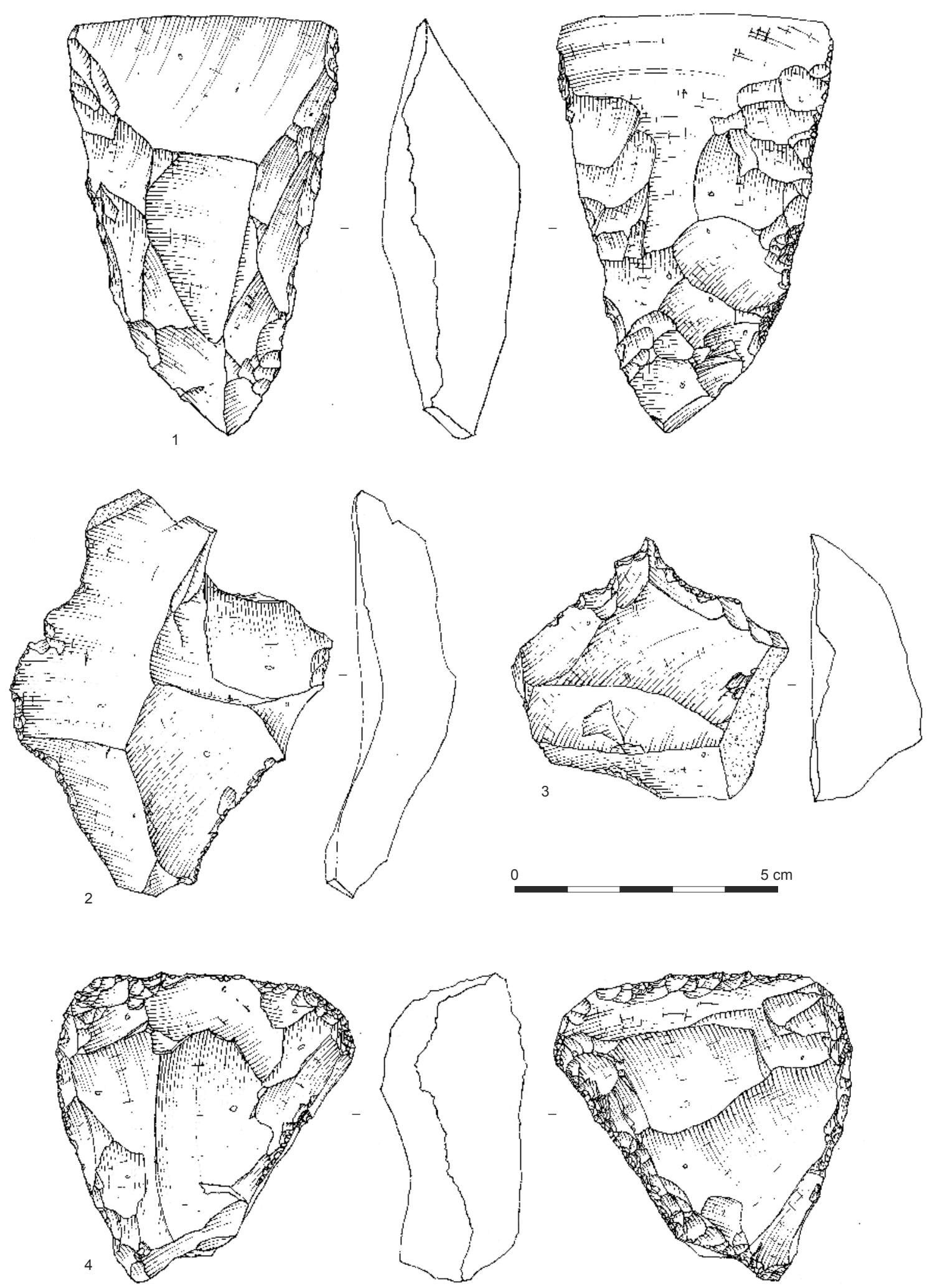

Figure 6 : Tilly-la-Campagne, structure 72 : mobilier lithique, outils sur éclat. 1 : tranchet bifacial; 2 : éclat épais écaillé/retouché; 3 : éclat épais denticulé; 4 : pièce esquillée. Dessins F. Charraud.

Figure 6: Tilly-la-Campagne, structure 72: lithic material, Flake tools. 1: bifacial tranchet; 2: reworked thick; 3: denticulated thick flake; 4: splintered piece. 
L'absence des grattoirs sur éclat et des armatures de projectiles est à remarquer mais elle n'est pas forcément étonnante si l'on considère que le lot étudié provient d'une unique structure, qui appartient probablement à un site plus étendu. La représentation ou l'absence de certaines classes d'objets n'est guère significative sur un effectif si réduit.

\section{BILAN DE L'OCCUPATION}

L'unique structure reconnue à Tilly-la-Campagne est vraisemblablement tout ce qui subsiste d'un site probablement plus étendu, arasé par les importantes activités agricoles de la Plaine de Caen. Les quelques éléments recueillis permettent de supposer que l'on a affaire à un site à vocation domestique. Par sa nature et la diversité du mobilier qu'elle contenait, cette fosse évoque celles qui bordent la plupart des architectures domestiques documentées pour le Néolithique ancien. La céramique comporte d'ailleurs comme principaux marqueurs culturels deux tessons décorés d'impressions au peigne, technique largement employée par les groupes culturels de tradition danubienne. Difficile d'être plus précis quant à cette attribution sauf à considérer la nature du mobilier en schiste, là encore caractéristique de la même ambiance chronoculturelle, et pour lequel la présence d'un fragment d'anneau à couronne large nous situerait à la fin de la période (Fromont, 2013). Malheureusement, aucune datation radio-isotopique ne peut étayer ces suppositions, la seule tentative s'étant soldée par un résultat aberrant.

Seul le mobilier en silex, de loin le plus abondant découvert dans cette structure, peut nous livrer quelques informations supplémentaires. Le petit panel des outils montre en effet une réelle diversité typologique et probablement fonctionnelle. La composition de cet ensemble est très classique si on la compare avec les autres assemblages du Villeneuve-SaintGermain régionaux, qui sont toujours très normés, avec des types d'outils très récurrents et identiques. L'ensemble de Tilly-la-Campagne ne remet pas en cause la typologie des assemblages de la région et constitue une occurrence supplémentaire d'un petit assemblage domestique dans la plaine jurassique de Normandie. En cela, il est comparable à plusieurs sites mis en évidence au cours des années 1990 et 2000 dans le Calvados, où de faibles effectifs mobiliers ont été retrouvés dans les restes arasés (une ou deux fosses à chaque fois) d'une occupation domestique : c'est le cas à Bernières-sur-Mer « La Crieux » (Hérard et Chanson, 2005), Saint-Manvieu-Norrey " Norrey " (Chancerel et al., 1993), Versainville « La Grande Haie " (Charraud et al., 2007) ou Éterville «Les Prés du Vallon » (Giazzon et al., 2009).

Cela dit, le mobilier découvert ne permet pas non plus de préciser l'attribution chronoculturelle, il donne un intervalle vaste concernant le Néolithique ancien d'obédience danubienne. La présence d'un tranchet pourrait signifier que le site se rapporte à la phase récente du B-VSG, si l'on se réfere à la représentation de ce type d'outils dans les contextes du Bassin parisien (Bostyn, 1994). Cela serait cohérent avec la présence d'un anneau à couronne large. On manque toutefois de référents fiables pour préciser l'évolution des tranchets dans le Grand Ouest (Marchand, 1999; Charraud, 2013).

La série lithique de Tilly-la-Campagne est relativement modeste mais elle permet d'observer, dans un contexte de découverte satisfaisant, un assemblage à vocation domestique proche des sources de silex du Cinglais. Qualitativement, le débitage laminaire est en tous points comparable aux ateliers $\mathrm{du}$ Cinglais ou aux autres sites du Néolithique ancien de la Plaine de Caen (ibid.). Toutes les étapes de la chaîne opératoire ne sont pas représentées. Le débitage laminaire a été effectué sur place à partir de rognons apportés déjà dégrossis. La qualité de la production et des matériaux suggère une origine minière de celui-ci. L'essentiel des supports laminaires a été taillé sur place. Rien ne permet de supposer l'apport de supports bruts ni, à l'inverse, de production excédentaire. Typologiquement, l'outillage est identique aux autres assemblages du Néolithique ancien. L'absence de certains types d'outils (armatures de projectiles ou grattoirs sur éclats) n'est pas significative compte tenu du la faiblesse représentative de l'échantillon observé.

Le site de Tilly constitue une occurrence supplémentaire, entérinant l'homogénéité des sites du Néolithique ancien de la Normandie, et à plus forte raison de l'ensemble du Bassin parisien pour cette période, ainsi que leur extension hors des vallées et à une certaine distance des cours d'eau.

\section{Bibliographie}

Allard P., 2005. L'industrie lithique des populations rubanées du Nord-Est de la France et de la Belgique. Internationale Archàologie band 86, VLM, Verlag Marie Leidorf GmbH. Rahden/Westf., 441 p.

Allard P., Bostyn F. et Martial E. avec la coll. de Collet H., FABRE J. et VALLIN L., 2010. « Les matières premières siliceuses exploitées au Néolithique moyen et final dans le Nord et la Picardie (France) ", in Billard C. et Legris M. (dir.), Premiers Néolithiques de l'Ouest. Cultures, réseaux, échanges des premières sociétés néolithiques à leur expansion, Colloque interrégional sur le Néolithique, Le Havre, 9-10 novembre 2007, Rennes, PUR, p. 117-162.

BAUdry A., 2012. Ressources animales et alimentation carnée à l'âge du Fer : le cas du Nord-Ouest de la France (Bretagne et BasseNormandie), Thèse de doctorat, université de Rennes 1, 385 p. 
Billard C., Bostyn F., Hamon C. et Meunier K., 2014. L'habitat du Néolithique ancien de Colombelles "Le Lazzaro " (Calvados), Mémoires de la Société préhistorique française, t. 58, Paris, éd. de la Société préhistorique française, 408 p.

Bostyn F., 1994. Caractérisation des productions et de la diffusion des industries lithiques du groupe néolithique de Villeneuve-SaintGermain, Thèse de doctorat de l'université de Paris X, 745 p.

Bostyn F. et Allard P., 2006. "Gestion de l'outillage en silex dans la culture de Villeneuve-Saint-Germain/Blicquy : du plus simple au plus complexe ", in Astruc L., Bon F., LÉA V., Milcent P.-Y. et Philibert S. (dir.), Normes techniques et pratiques sociales. De la simplicité des outillages pré-et protohistoriques. XXVI' rencontres internationales d'archéologie et d'histoire d'Antibes, éd. APDCA, Antibes, p. 195-206.

Chancerel A., Desloges J., Dron J.-L. et San Juan G., 1992. "Le début du Néolithique en Basse-Normandie ", in Le Roux C.-T. (dir.), Paysans et bâtisseurs : l'émergence du Néolithique atlantique et les origines du mégalithisme, Actes du $17^{e}$ colloque interrégional sur le Néolithique (Vannes, 29-31 octobre 1990), Revue archéologique de l'Ouest, suppl. n 5, p. 153-173.

Chancerel A., Marcigny C. et Ghesquiere E. (dir.), 2006. Le plateau de Mondeville (Calvados) du Néolithique à l'àge du Bronze, Paris, Éd. de la Maison des sciences de l'homme, DAF, $\mathrm{n}^{\circ}$ 99, $208 \mathrm{p}$.

Chancerel A., Forfait N., Ghesquiere E. et Lepaumier H., 1993. "Un silo du groupe de Villeneuve-Saint-Germain à Saint Manvieu-Norrey (Calvados) », in Chancerel A. (dir.), Projet collectif de recherches sur le Néolithique moyen en BasseNormandie, Rapport d'activité, $2{ }^{e}$ année de recherche, 1993, SRA Basse-Normandie, 6 p.

Charraud F. avec la coll. de Fromont N., Hulin G., Lemee M., Bohard B., Dron J.-L. et Quevillon S., 2007. Versainville (Calvados) "La Grande Haie ", Rapport de fin d'opérations, prospections géophysiques et sondages archéologiques, $119 \mathrm{p}$.

Charraud F., 2009. L'acquisition et la transformation initiale $d u$ silex du Cinglais dans la plaine de Caen à la préhistoire récente, Rapport de prospection thématique, SRA Basse-Normandie, Caen, 103 p.

Charraud F., 2013. Espaces interculturels et évolution des systèmes techniques au Néolithique dans le Nord-Ouest de la France. Productions, usages et circulation des outillages en silex jurassiques de Normandie, Thèse de doctorat de l'université Nice-Sophia Antipolis, 2 vol., 480 et 538 p.

Coutard S., 1998. Panorama des silex jurassiques bas-normands. Essai de caractérisation et répartition, Mémoire de maîtrise de l'université de Caen, département de géologie, 83 p.

Fromont N., 2013. Anneaux et cultures du Néolithique ancien. Production, circulation et utilisation entre massifs ardennais et armoricain, BAR International Series 2499, Oxford, 682 p.

Germain-Vallee C., Jan D., Charraud F., Fromont N., Hamon C., Riquier C., Beguier I. et Marcoux N., 2014. « Le site
Villeneuve-Saint-Germain de Verson "Les Mesnils" (Calvados, Basse-Normandie) : présentation liminaire ", in INTERNEO 10 - 2014, Journée d'information du 22 novembre 2014, Paris, éd. de la Société préhistorique française, p. 143-152.

Germain-Vallée C. et Lespez L., 2011. « L'apport des recherches géomorphologiques et micro-morphologiques récentes à l'archéologie des paysages de la plaine de Caen (Calvados, BasseNormandie) ", in Lespez L. (dir.), Géoarchéologie dans l'ouest de la France, Norois, n 220-2011/3, 143-178.

Ghesquiere E., Marcigny C., Desloges J. et Charraud F., 2008. "La production de lames en silex bathonien dans la plaine de Caen : redécouverte de la minière des Longrais à Soumont-Saint-Quentin (Calvados) ", in INTERNEO 7 2008, Journée d'information du 22 novembre 2008, Paris, éd. de la Société préhistorique française, p. 103-120.

Giazzon D., 2009. Eterville (Calvados) "Les Prés du Vallon ", Rapport final de fouille, Inrap, Direction interrégionale Grand-Ouest, Cesson-Sévigné, 240 p.

Giraud P., Juhel L., Hamon C., Germain C., Savary X. et JAN D., 2012. L'habitat du Néolithique ancien de Fontenay-leMarmion "Le Grand Champ/Le chemin Haussé " (Calvados), Revue archéologique de l'Ouest, $\mathrm{n}^{\circ}$ 29, p. 51-87.

Guilbert R., Philibert S. et Peresani M., 2006. "L'outil expédient : une partie intégrante de la production mésolithique ", in Astruc L., Bon F., Léa V., Milcent P.-Y. et Philibert S. (dir.), Normes techniques et pratiques sociales. De la simplicité des outillages pré- et protohistoriques. XXVIe rencontres internationales d'archéologie et d'histoire d'Antibes, Antibes : éd. APDCA, p. 161-172.

Herard B. et Chanson K., 2005. Bernières-sur-Mer (Calvados) "La Crieux ", Rapport de diagnostic archéologique. SRA Basse-Normandie, non paginé.

JAN D., 2010. Les céramiques néolithiques à dégraissant végétal en Basse-Normandie: Etude des pâtes, identification des végétaux et protocole expérimental, Vol.1 Elaboration du corpus, étude pétrographique des pâtes et observation des inclusions végétales, Mémoire de master 1 "Archéologie et environnement ", université Paris $1,94 \mathrm{p}$.

JAN D., 2011. Les céramiques néolithiques à dégraissant végétal en Basse-Normandie: Etude des pâtes, identification des végétaux et protocole expérimental, Vol.2 Protocole expérimental et identification du dégraissant végétal, Mémoire de master 2 "Archéologie et environnement ", université Paris 1, 88 p.

Marchand G., 1999. La néolithisation de l'Ouest de la France : caractérisation des industries lithiques, Oxford, BAR International Series 748, $381 \mathrm{p}$.

Marcigny C. (dir.), 2010. Écouché (Orne) "Carrière MEAC ", Rapport final d'opération de fouille préventive, SRA BasseNormandie, $96 \mathrm{p}$.

Pelegrin J., 1995. "Réflexions méthodologiques sur l'étude de séries lithiques en contexte d'atelier ou de mines ", in Pelegrin 
J. et Richard A. (dir.), Les mines de silex au Néolithique en Europe : avancées récentes, Actes de la table ronde internationale de Vesoul, 18-19 octobre 1991, Paris, CTHS, p. 159-166.
Petrequin P. et Petrequin A.-M., 1993. Écologie d'un outil : la hache de pierre en Irian Jaya (Indonésie), monographie du CRA, $\mathrm{n}^{\circ} 14$, éd. du CNRS, Paris, 444 p.

Zusammenfassung: Tilly-la-Campagne (Calvados, Normandie, France) „Chemin RN 158“, Neue Hinweise auf eine Besiedlung im Frühneolithikum - Eine archäologische Baggerprospektion, die 2007 vom Archäologischen Dienst des Departements Calvados bei Tilly-la-Campagne (Calvados, Normandie, France) durchgeführt wurde, hat es gestattet, einen isolierten Befund des Frühneolithikums (Befund 72) freizulegen. Es handelt sich dabei um eine in zwei Loben unterteilte Grube von geringer Tiefe und von unregelmäßigem Umriss, die in den anstehenden Löss eingetieft war. Die Grube war Ost-West ausgerichtet und maß 4,40 m X2,15 m. Das geborgene Fundmaterial, das in erster Linie aus dem östlichen Teil der Grube geborgen wurde, war nicht sehr umfangreich, umfasste jedoch ganz unterschiedliches Fundgut. Es handelt sich um lithisches Fundmaterial (587 Funde), Keramik (199 Keramikscherben), einige wenige Fragmente von Mahlsteinen (14 Objekte), Fragmente von Armringen aus Tonschiefer (4 Funde), einige rundliche Ausbildungen oolitischen Hämatits (5 Funde), archäozoologische Reste (229 Knochen) sowie einige verkohlte Haselnussschalen. Eine 14C-Datierung wurde an letzteren versucht, jedoch ohne ein brauchbares Ergebnis zu liefern. Dieser Befund stellt vermutlich alles dar, was von einem ursprünglich größeren Fundplatz übrig geblieben ist, der durch die umfangreiche landwirtschaftliche Nutzung im Becken von Caen weitgehend erodiert wurde. Einige der geborgenen Funde lassen vermuteten, dass es sich um einen Siedlungsfundstelle gehandelt hat. Die charakteristischen Eigenschaften der Grube sowie das aus ihrer Verfüllung geborgene Fundmaterial erlauben es, sie an die Seite jener Gruben zu stellen, die als Längsgruben die frühneolithischen Häuser begleiteten. Das keramische Fundmaterial enthielt als typische kulturelle Indikatoren zwei kammstrichverzierte Scherben, einer Technik, die in den Kulturgruppen vorkommt, die in bandkeramischer Tradition stehen. Das Auftreten eines Fragmentes eines breiten Scheibenringes sowie eines Dechsels verweist insbesondere auf die Kulturgruppe von Blicquy/Villeneuve-Saint-Germain récent.

Schlüsselwörter: Frühneolithikum, Normandie, Calvados, Ebene von Caen, Villeneuve-Saint-Germain, Siedlung, Graben, Steingeräte, Schmuck aus Tonschiefer, Keramik, archäozoologische Funde, Hämatit, Flint, makrolithische Steingeräte. 